\title{
Modification of Serum Galectin-3 and Reversal of Cardiac Remodeling Following Pediatric Transcatheter Atrial Septal Defect Closure
}

\author{
Omnia Shafei ${ }^{1,2, *}$, Alyaa A. Kotby ${ }^{1,2}$, Mona M. El Ganzoury ${ }^{1,2}$, Nevin M. Habeeb ${ }^{1,2}$ and Eman Elsayed ${ }^{1,2}$ \\ ${ }^{1}$ Department of Pediatrics, Faculty of Medicine, Ain Shams University, Cairo, Egypt \\ ${ }^{2}$ Children's Hospital, Cardiology Unit, Ain Shams University Hospitals, Cairo, Egypt \\ ${ }^{\star}$ Corresponding Author: Omnia Shafei. Email: omniashafy@gmail.com
}

Received: 17 April 2021 Accepted: 30 June 2021

\begin{abstract}
Objectives: We aimed to evaluate the effect of percutaneous atrial septal defect (ASD) closure in children using right heart indices and serum galectin-3 (Gal-3), as potential biomarkers of right heart remodeling. Methods: This case-control prospective study included 40 children with ASD and 25 control subjects. An echocardiographic evaluation was performed before the procedure, as well as 24 h, 1 month, and 6 months after intervention. Serum Gal-3 was measured before, and 1 month after the procedure. Results: Serum Gal-3 concentration, right atrial (RA) dimensions, right ventricular (RV) dimensions, indexed RA area, and right index of myocardial performance (RIMP) were significantly increased in children with ASD compared with control subjects while tricuspid annular plane systolic excursion (TAPSE) was significantly decreased. Six months after closure, RA, and RV dimensions significantly decreased and RV function improved (RIMP decreased and TAPSE increased). Gal-3 oncentration significantly decreased 1 month after ASD closure, but it did not reach normal range compared with control subjects. A positive correlation between Gal-3 and age at closure, RA area, RV dimensions, and RIMP was observed. A positive correlation was observed between the decrease in Gal-3 concentration and the decrease in RA area and RV dimensions 1 month after ASD closure. A significant negative correlation was observed between TAPSE and Gal-3 concentration before and after intervention. Conclusions: Percutaneous ASD closure can improve right-sided indices and decrease serum Gal-3 concentration. Gal-3 can be used as a sensitive biomarker of right heart remodeling, with a decrease in Gal-3 concentration suggesting reversal of maladaptive remodeling.
\end{abstract}

\section{KEYWORDS}

Percutaneous device closure; Galectin-3; cardiac remodeling; children; atrial septal defect

\section{Introduction}

Atrial septal defect (ASD) affects 1.64 per 1000 live births and it is considered one of the most common congenital heart diseases [1]. ASD is a common cause of chronic right-sided heart volume overload. Longstanding left-to-right shunt can trigger adverse cardiac remodeling which is defined as a change in the structure and function of the heart. Cardiac remodeling involves right heart dilatation with development of right heart failure, atrial arrhythmia, and high pulmonary vascular resistance (PVR) [2-4]. 
Percutaneous device closure is the treatment of choice for most cases of ASD [5,6]. Closure of ASD results in an improvement in right heart dimensions and function secondary to a change in cardiac hemodynamics [7].

Blood-based biomarkers could provide insight into heart remodeling after ASD closure and predict myocardial inflammation and fibrosis [8]. Serum Galectin-3 (Gal-3) is a multifunctional protein ( $\beta$ galactosidase-binding lectin) secreted by cardiac macrophages. Gal-3 plays an integral role in myocardial remodeling as it is involved in both physiological and pathological processes, including myofibroblast proliferation, cell growth, inflammation, and matrix deposition [9]. Gal-3 promotes oxidative stress in human cardiac fibroblasts which results in cardiac damage [10].

Gal-3 is a promising biomarker in children with heart disease. It has been used as a biomarker of disease severity in pediatric patients with left-sided heart failure with either a normal or reduced ejection fraction [11]. The concentration of Gal-3 could predict the pathophysiology of cardiac failure and its progression as well as pulmonary hypertension $[12,13]$.

Serum Gal 3 concentration correlates with right heart systolic function and right-sided dilatation in adults, since it is secreted by right ventricular (RV) macrophages in response to a change in pulmonary pressure, which results in right heart adaptation in response to elevated pressure [14]. Moreover, a correlation between serum Gal-3 and fibrosis in the right atrium (RA) was observed where a high serum Gal-3 concentration indicated atrial remodeling [15]. The role of serum Gal-3 in children with ASD has not been fully studied. The present study evaluated the effect of percutaneous ASD closure in children using right heart echocardiographic parameters and serum Gal-3 concentration, as a potential biomarker of right heart remodeling.

\section{Patients and Methods}

This was a single-center prospective interventional case-control study conducted at the Pediatric Cardiology Unit, Children's Hospital, Ain Shams University. Forty children and adolescents with secundum ASD and 25 healthy age- and sex-matched children were enrolled between December 2018 and June 2020. The study protocol was approved by the ethics committee of the Faculty of Medicine, Ain Shams University.

Children were selected according to the inclusion criteria: secundum ASD with a pulmonary/systemic flow ratio $(\mathrm{Qp} / \mathrm{Qs}) \geq 1.5: 1$ and symptoms of right-sided overload (dyspnea, feeding intolerance, or reduced exercise capacity); an ASD size of $>5 \mathrm{~mm}$ and a rim of $>5 \mathrm{~mm}$ from the ASD to the nearby structure (superior vena cava, pulmonary veins, atrioventricular valves, inferior vena cava). The exclusion criteria were primum ASD or sinus venosus defect, associated other congenital heart disease, PVR $>2 / 3$ systemic vascular resistance/PVR of $>5$ Wood units, and right-to-left atrial shunt. Written informed consent was obtained from the parents of children enrolled in the study.

Detailed history and clinical data were obtained, with special emphasis on cardiac symptoms, atrial arrhythmias, current medications, anthropometric measurement, oxygen saturation, and signs of heart failure. Chest X-ray and 12-lead electrocardiography were performed as standard preoperative assessments.

Serum Gal-3 concentration was measured using an enzyme-linked immunosorbent assay (ELISA) (Quantikine Human Galectin-3 Immunoassay catalog No. DGAL3). Samples were collected from all children via phlebotomy before transcatheter device closure and repeated 1 month after intervention. Samples were centrifuged and stored at $-20^{\circ} \mathrm{C}$. No other blood biomarkers were measured.

Transthoracic echocardiography (TTE) was performed for all children using the Vivid E9 machine (GE Vingmed Ultrasound N-3191, Horton, Norway). Children with ASD were examined before the procedure, as well as $24 \mathrm{~h}, 1$ month, and 6 months after intervention. ASD and right chambers assessments were performed according to established criteria from the European and American Society of Echocardiography 
[16-20]. The Qp/Qs ratio was estimated by Doppler measurements of pulmonary and aortic stroke volume [17]. RA dimensions were assessed by RA major and minor diameters while RA area was estimated by planimetry "Figs. 1A-1C". RV dimensions in the form of RV mid-cavity, RV longitudinal, and RV basal diameters were measured. RV function was assessed using right index of myocardial performance (RIMP) by tissue Doppler (TDI) and tricuspid annular plane systolic excursion TAPSE "Figs. 2A and 2B". RV systolic pressure (RVSP) and mean pulmonary artery pressure (PAP) were measured. RA volume was calculated as follow: $8 / 3 \pi$ (RA area $^{2} /$ RA major length) $[16,19]$. RA pressure was estimated by inferior vena cava (IVC) diameter and inferior vena cava collapsibility index (IVCCI) in a subcostal view.
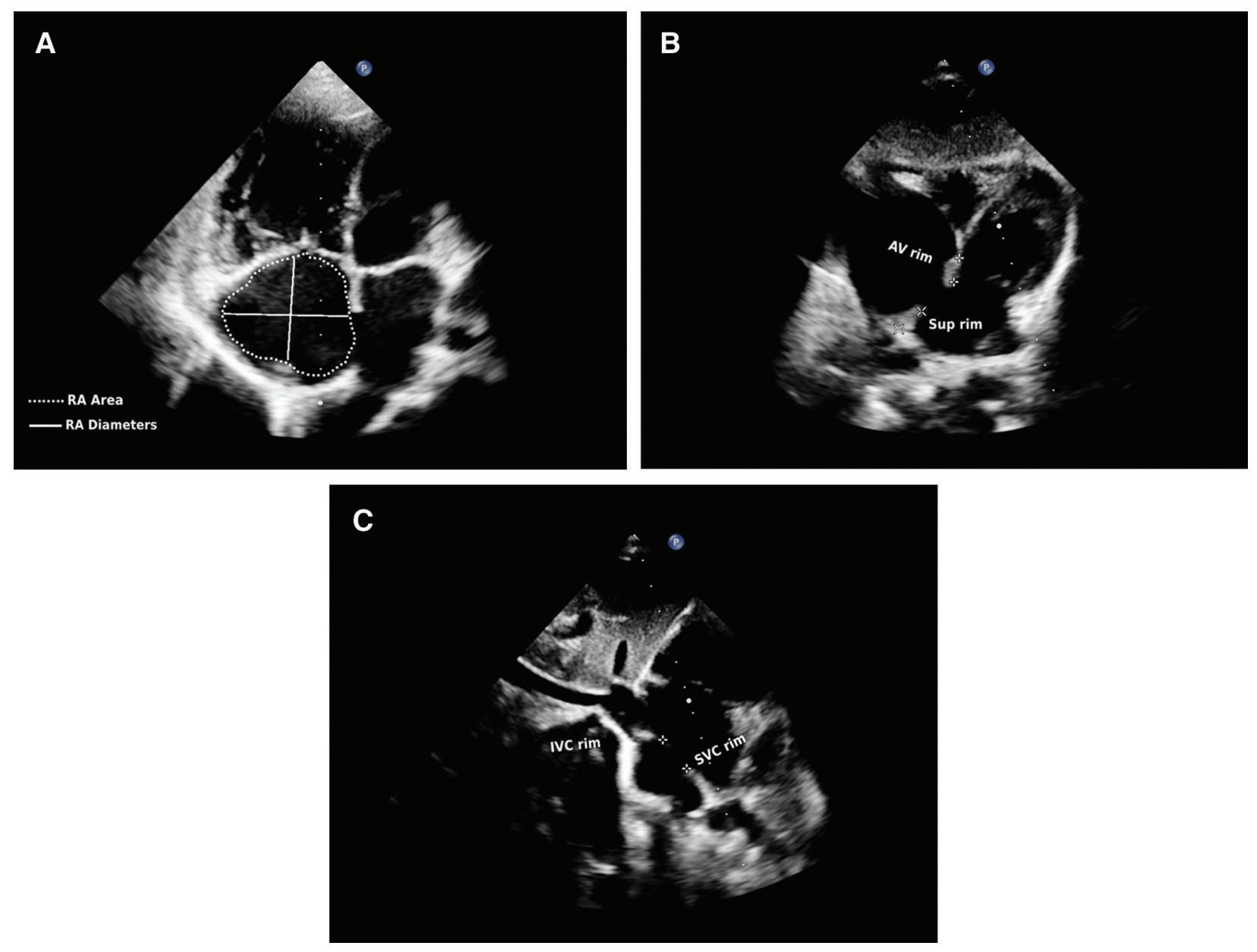

Figure 1: (A) RA area, RA major, and minor diameters of a studied child from TTE apical-4 chamber view. (B and C) ASD size, and rims from TTE subcostal views

Transesophageal echocardiography (TEE) was performed under general anesthesia using the CX50 Philips ultrasound machine with X7-2t TEE probe for children with a body weight of $>35 \mathrm{~kg}$ or the Affiniti 50 ultrasound machine with S7-3t TEE probe for children with a body weight of $<35 \mathrm{~kg}$ (Philips Medical Systems, Andover, MA, USA). Multiple TEE views were used to assess the total interatrial septum length, ASD morphology, maximal ASD diameter, ASD rims, device position, and any residual shunt after device closure. Transcatheter device closure of ASD was performed under both echocardiographic and fluoroscopic guidance. A device 2-3 mm larger than the ASD diameter was selected [5]. However, if the aortic rim was deficient or the rims were floppy, a device $4 \mathrm{~mm}$ larger than 
the maximal diameter was selected. Two types of devices were used as regular septal occluders for oval defects and the Amplatzer cribriform septal occlude was used in case of a fenestrated septum and its size was determined by total septal length. In one patient, there were two defects, and the intervening tissue was $7 \mathrm{~mm}$, so two devices were placed (one for each defect) "Figs. 3A and 3B".
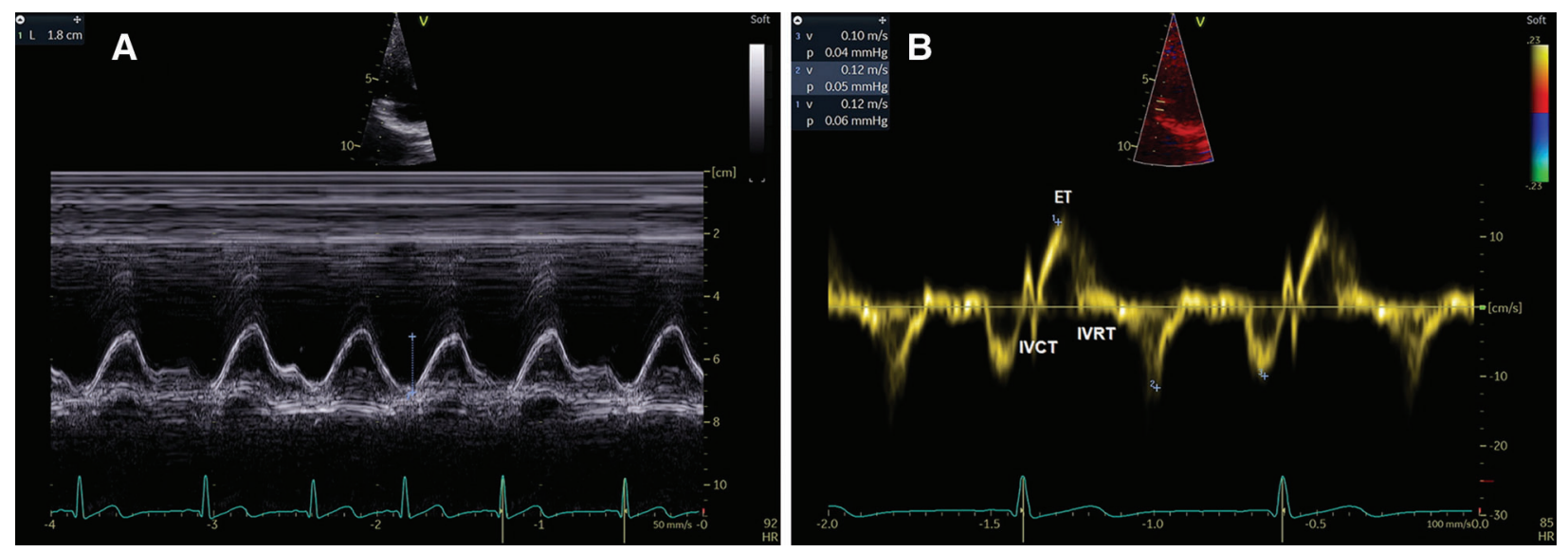

Figure 2: (A) Tricuspid annular plane systolic excursion (TAPSE). (B) Right index of myocardial performance by tissue Doppler (RIMP-TDI) (ET; Ejection time, IVCT; isovolumetric contraction time, IVRT; isovolumetric relaxation time) from TTE apical views


Figure 3: (A) TEE mid-esophageal short-axis view of a 3-year-old child with two ASDs where one was closed using a device and the other was shunting. (B) TEE four-chamber view showing two devices wellseated in place with no residual shunt

\section{Statistical Analysis}

Data were analyzed using the Statistical Package for Social Sciences (SPSS), version 23 (IBM Co., Armonk, NY, USA). Quantitative variables are expressed as mean \pm standard deviation. Comparisons of quantitative data between patients and controls were performed using the independent t-test. The paired t-test was used to compare between two paired groups, while repeated-measures analysis of variance was used to compare more than two paired groups. The correlations between Gal-3 concentration and patients' characteristics were assessed using Spearman's correlation coefficient. The sample size was 
adequate to test the hypothesis; the required sample size was 25 using the $\mathrm{G}^{*}$ Power program with an alpha error of 5\%, a power of $80 \%$, and the size of variation of Gal-3 represented by an effect size of 0.6 . A $p$-value $<0.05$ was considered statistically significant.

\section{Results}

This study included 40 children with ASD (17 males and 23 females) and 25 healthy children (10 males and 15 females) as the control group. Of the 40 study patients, 38 patients underwent transcatheter ASD device closure and completed 6 months of follow up, while 2 patients were referred for surgery after TEE due to insufficient rims. PVR in Wood units ranged from 0.87 to 1.09 , and Qp/Qs ranged from 1.7 to 5.3. The mean maximal ASD diameter was $11.48 \pm 2.88 \mathrm{~mm}$. The mean total septal length was $30.25 \pm$ $2.92 \mathrm{~mm}$. Twenty-five patients had one ASD defect, 8 patients had two defects and 7 patients had a fenestrated septum. ASD was successfully closed in 37 patients with one device, except for one patient in whom, two devices were needed to close the defect.

Demographic data, echocardiographic parameters, and Gal-3 concentration are shown in Table 1. In terms of clinical data, $57.5 \%$ of patients suffered from dyspnea on exertion, $32.5 \%$ suffered from palpitations, $10 \%$ developed recurring chest infection, and one patient developed decompensated heart failure.

Table 1: The demographic data, echocardiographic parameters, and Gal-3 concentration before intervention

\begin{tabular}{|c|c|c|c|}
\hline & $\begin{array}{l}\text { Control group } \\
\mathrm{n}=25 \\
\text { Mean } \pm \mathrm{SD} \\
\text { Range }\end{array}$ & $\begin{array}{l}\text { Patient group } \\
\mathrm{n}=40 \\
\text { Mean } \pm \mathrm{SD} \\
\text { Range }\end{array}$ & $p$-value \\
\hline $\begin{array}{l}\text { Female } \\
\text { Male }\end{array}$ & $\begin{array}{l}15(60.0 \%) \\
10(40.0 \%)\end{array}$ & $\begin{array}{l}23(57.5 \%) \\
17(42.5 \%)\end{array}$ & $0.842 *$ \\
\hline Age in years & $\begin{array}{l}6.83 \pm 4.52 \\
2-16\end{array}$ & $\begin{array}{l}6.07 \pm 3.74 \\
2.1-16\end{array}$ & $0.463 \bullet$ \\
\hline BMI $\left(\mathrm{kg} / \mathrm{m}^{2}\right)$ & $\begin{array}{l}16.80 \pm 4.00 \\
0.7-21.4\end{array}$ & $\begin{array}{l}17.05 \pm 2.80 \\
12.24-24.49\end{array}$ & $0.769 \bullet$ \\
\hline BSA & $\begin{array}{l}0.90 \pm 0.35 \\
0.52-1.64\end{array}$ & $\begin{array}{l}0.76 \pm 0.23 \\
0.44-1.35\end{array}$ & $0.049 \bullet$ \\
\hline RA minor dimension $(\mathrm{cm})$ & $\begin{array}{l}2.48 \pm 0.45 \\
1.8-3.3\end{array}$ & $\begin{array}{l}3.02 \pm 0.45 \\
2.3-4.2\end{array}$ & $<0.001 \bullet$ \\
\hline RA major dimension $(\mathrm{cm})$ & $\begin{array}{l}2.90 \pm 0.51 \\
1.9-3.8\end{array}$ & $\begin{array}{l}3.53 \pm 0.60 \\
2.4-4.9\end{array}$ & $<0.001 \bullet$ \\
\hline Indexed RA Area $\left(\mathrm{cm}^{2} / \mathrm{m}^{2}\right)$ & $\begin{array}{l}7.67 \pm 0.97 \\
5.33-9.2\end{array}$ & $\begin{array}{l}13.50 \pm 3.11 \\
7.5-24.08\end{array}$ & $<0.001 \bullet$ \\
\hline Indexed RA Volume (mL/m²) & $\begin{array}{l}14.84 \pm 3.27 \\
9.51-20\end{array}$ & $\begin{array}{l}32.85 \pm 12.77 \\
11.93-71.72\end{array}$ & $<0.001 \bullet$ \\
\hline IVCCI (\%) & $\begin{array}{l}62.02 \pm 9.16 \\
40-80\end{array}$ & $\begin{array}{l}42.49 \pm 8.90 \\
25-57.14\end{array}$ & $<0.001 \bullet$ \\
\hline RV Mid Cavity dimension (cm) & $\begin{array}{l}2.34 \pm 0.44 \\
1.7-3.3\end{array}$ & $\begin{array}{l}2.80 \pm 0.58 \\
1.9-4.00\end{array}$ & $0.001 \bullet$ \\
\hline
\end{tabular}




\begin{tabular}{llll}
\hline Table 1 (continued). & & & \\
& Control group & Patient group & $p$-value \\
& $\mathrm{n}=25$ & $\mathrm{n}=40$ & \\
& Mean $\pm \mathrm{SD}$ & Mean $\pm \mathrm{SD}$ & \\
& Range & Range & $0.001 \bullet$ \\
& $2.84 \pm 0.60$ & $3.38 \pm 0.64$ & \\
\hline RV basal & $2.1-3.8$ & $2.3-4.7$ & $0.006 \bullet$ \\
Dimension $(\mathrm{cm})$ & $4.97 \pm 1.10$ & $5.69 \pm 0.94$ & $<0.001 \bullet$ \\
RV Longitudinal dimension $(\mathrm{cm})$ & $3.5-7.2$ & $4.3-7.4$ & \\
& $15.88 \pm 3.41$ & $43.70 \pm 6.02$ & $<0.001 \bullet$ \\
RVSP (mmHg) & $11-22$ & $32-55$ & \\
Mean PAP (mmHg) & $4.45 \pm 0.89$ & $10.39 \pm 2.02$ & $0.005 \bullet$ \\
TAPSE (cm) & $3.2-6.5$ & $6.7-16$ & \\
& $2.53 \pm 0.27$ & $2.32 \pm 0.29$ & $<0.001 \bullet$ \\
RIMP & $2.1-3.1$ & $1.8-2.9$ & $<0.001 \bullet$ \\
Gal-3 concentration $(\mathrm{ng} / \mathrm{ml})$ & $0.30 \pm 0.06$ & $0.38 \pm 0.07$ & \\
\hline
\end{tabular}

Note: • :Independent t-test; *: Chi-square test.

BMI; body mass index, BSA; body surface area, RA; right atrium, RV; right ventricle, IVCCI; inferior vena cava collapsibility index, RVSP; right ventricular systolic pressure, PAP; pulmonary artery pressure, TAPSE; Tricuspid annular plane systolic excursion, RIMP; RV myocardial performance index, Gal-3; Galectin-3.

Statistically significant increases in RA dimensions, indexed RA area and volume, RVSP, and RV dimensions (RV mid-cavity, RV basal and RV longitudinal dimension) were observed before intervention in the studied patients compared with control subjects. Impaired RV performance was revealed by significantly higher RIMP and lower TAPSE values which were observed in patients with ASD before intervention. The mean size of the device used for ASD closure was $19.62 \pm 6.88 \mathrm{~mm}$ (range: 12 to $35 \mathrm{~mm}$ ). Thirty-one patients received the regular septal occluder and 7 patients received the Amplatzer cribriform septal occluder. Three of the studied patients received clopidogrel treatment $(1 \mathrm{mg} / \mathrm{kg} / \mathrm{day})$ in addition to salicylate therapy (3-5 $\mathrm{mg} / \mathrm{kg} /$ day) as they had large devices.

There was a statistically significant decrease in RA and RV indices during the 6-month follow up after device closure (Table 2). One month after ASD closure, no significant difference was observed between patients and control subjects with regard to RV mid-cavity dimension ( $p 0.243$ ), RV longitudinal dimension ( $p$ 0.127), RV basal dimension ( $p$ 0.191), TAPSE ( $p$ 0.529), and RIMP ( $p$ 0.147). By the end of 6-month follow up, no significant difference was observed between patients and control subjects regarding RA major dimension ( $p$ 0.187), RA minor dimension ( $p$ 0.459), and RA pressure estimated by IVCCI ( $p$ 0.931). However, there was a significant difference between patients and control subjects in indexed RA area and indexed RA volume at 6-months after closure $(p<0.001)$.

The mean Gal-3 concentration and Gal-3 concentration range were significantly higher in patients before device closure (mean: $3.71 \mathrm{ng} / \mathrm{ml}$, range: $2.5-6 \mathrm{ng} / \mathrm{ml}$ ) compared with control subjects (mean: $0.63 \mathrm{ng} / \mathrm{ml}$, range: $0.45-1 \mathrm{ng} / \mathrm{ml} ; p<0.001$ ). The receiver operating characteristic (ROC) curve of Gal-3 concentration revealed that a cut-off value of $1 \mathrm{ng} / \mathrm{ml}$ was discriminative between patients and control subjects with $100 \%$ specificity and sensitivity "Fig. 4" and Table 3. Gal-3 concentration significantly 
decreased after ASD closure; however, Gal-3 concentration was still significantly higher in studied patients compared with control subjects 1 month after ASD closure $(p<0.001)$.

Table 2: Echocardiographic parameters and serum Gal-3 concentration before and after intervention

\begin{tabular}{llllll}
\hline $\begin{array}{l}\text { Echocardiographic Parameters/ } \\
\text { Biomarker level } \\
\text { (Mean } \pm \text { SD) }\end{array}$ & Before & 24-hours & 1 month & 6 months & $p$-value \\
\hline $\begin{array}{l}\text { Indexed RA Area } \\
\left(\mathrm{cm}^{2} / \mathrm{m}^{2}\right)\end{array}$ & $13.50 \pm 3.11$ & $13.01 \pm 3.05$ & $11.89 \pm 2.98$ & $10.61 \pm 2.77$ & $<0.001 \bullet$ \\
RA minor dimension (cm) & $3.02 \pm 0.45$ & $2.92 \pm 0.48$ & $2.74 \pm 0.48$ & $2.57 \pm 0.49$ & $<0.001 \bullet$ \\
RA Major dimension (cm) & $3.53 \pm 0.60$ & $3.46 \pm 0.59$ & $3.26 \pm 0.56$ & $3.10 \pm 0.59$ & $<0.001 \bullet$ \\
Indexed & $32.85 \pm 12.77$ & $30.82 \pm 12.53$ & $27.19 \pm 11.58$ & $22.70 \pm 9.96$ & $<0.001 \bullet$ \\
RA Volume (mL/m $\left.{ }^{2}\right)$ & & & & & \\
IVCCI (\%) & $42.49 \pm 8.90$ & $49.77 \pm 7.10$ & $53.34 \pm 7.00$ & $61.84 \pm 7.95$ & $<0.001 \bullet$ \\
RV Mid Cavity diameter (cm) & $2.80 \pm 0.58$ & $2.68 \pm 0.58$ & $2.48 \pm 0.50$ & $2.34 \pm 0.45$ & $<0.001 \bullet$ \\
RV basal diameter (cm) & $3.38 \pm 0.64$ & $3.31 \pm 0.68$ & $3.05 \pm 0.64$ & $2.93 \pm 0.64$ & $<0.001 \bullet$ \\
RV Longitudinal diameter (cm) & $5.69 \pm 0.94$ & $5.62 \pm 0.95$ & $5.37 \pm 0.94$ & $5.37 \pm 0.94$ & $<0.001 \bullet$ \\
RVSP (mmHg) & $43.70 \pm 6.02$ & $36.84 \pm 5.66$ & $30.63 \pm 6.36$ & $23.63 \pm 6.75$ & $<0.001 \bullet$ \\
TAPSE & $2.42 \pm 0.29$ & $2.44 \pm 0.25$ & $2.48 \pm 0.31$ & $2.55 \pm 0.39$ & $<0.001 \bullet$ \\
RIMP & $0.38 \pm 0.07$ & $0.38 \pm 0.06$ & $0.33 \pm 0.06$ & $0.29 \pm 0.08$ & $<0.001 \bullet$ \\
Gal-3 level (ng/ml) & $3.71 \pm 0.80$ & - & $2.13 \pm 0.66$ & - & $<0.001 *$ \\
\hline
\end{tabular}

Note: $\bullet$ Repeated Measures ANOVA; *Paired $t$-test.

Table 3: Diagnostic performance of Gal-3 concentration in discrimination between patients and controls

\begin{tabular}{llllll}
\hline Cut off point & AUC & Sensitivity & Specificity & PPV & NPV \\
\hline$>1 \mathrm{ng} / \mathrm{ml}$ & 1.000 & 100.00 & 100.00 & 100.0 & 100.0 \\
\hline
\end{tabular}

Note: AUC; area under the curve, PPV; positive predictive value, NPV; negative predictive value.

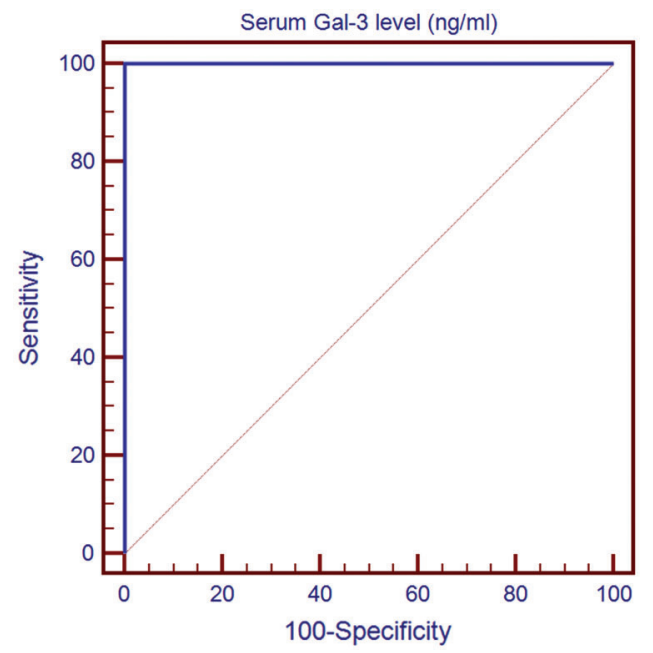

Figure 4: The ROC curve of serum Gal-3 concentration $(\mathrm{ng} / \mathrm{ml})$ in discrimination between patients and control subjects 
Gal-3 concentration positively correlated with the age of patients in the control group $(\mathrm{r}=0.727, p<0.001)$. Gal-3 concentration in the study group positively correlated with age at closure $(\mathrm{r}=0.519, p=0.001)$, ASD size $(\mathrm{r}=$ $0.442, p=0.004)$, indexed RA area $(\mathrm{r}=0.571, p<0.001)$, indexed $\mathrm{RA}$ volume $(\mathrm{r}=0.419, p=0.007)$, RV basal diameter ( $\mathrm{r}=0.681, p<0.001)$, RVSP $(\mathrm{r}=0.421, p=0.007)$, and RIMP $(\mathrm{r}=0.675, p<0.001)$, and negatively correlated with TAPSE $(\mathrm{r}=-0.510, p=0.001)$ "Fig. 5". A positive correlation was observed between the changes that occurred in Gal-3 concentration (median [interquartile range]: -1.5 [ -2 to -1.1$]$; range: -4.2 to 0.3 ) and the changes that occurred in the following parameters after 1 month from ASD closure, indexed RA area $(\mathrm{r}=0.488, p=0.002)$, indexed RA volume $(\mathrm{r}=0.409, p=0.011)$, $\mathrm{RV}$ basal diameter $(\mathrm{r}=0.559, p<0.001)$, $\operatorname{RVSP}(\mathrm{r}=0.519, p=0.001)$, and RIMP $(\mathrm{r}=0.746, p<0.001)$. A negative correlation was observed between Gal-3 concentration and the changes in TAPSE $(\mathrm{r}=-0.557, p<0.001)$. However, Gal-3 concentration did not correlate with body mass index $(p=0.670)$, RA diameter $(p=0.838)$, or RV longitudinal dimension $(p=0.420)$.

With a univariate analysis, Gal-3 concentration positively correlated with age at closure $(p=0.002)$, ASD size $(p=0.001)$, indexed RA area $(p<0.001)$, indexed RA volume $(p=0.007)$, RV basal diameter $(p<0.001)$, RVSP $(p=0.004)$, and RIMP $(p<0.001)$, and negatively correlated with TAPSE $(p<$ $0.001)$. In addition, the decrease in serum Gal-3 concentration correlated with the decrease in indexed RA area $(p<0.001)$, indexed RA volume $(p<0.001)$, RV basal diameter $(p<0.001)$, RVSP $(p<0.001)$, and RIMP $(p<0.001)$, and negatively correlated with the increase in TAPSE $(p<0.001)$. In a multivariate analysis, Gal-3 concentration was highly associated with age at closure $(p=0.030)$.
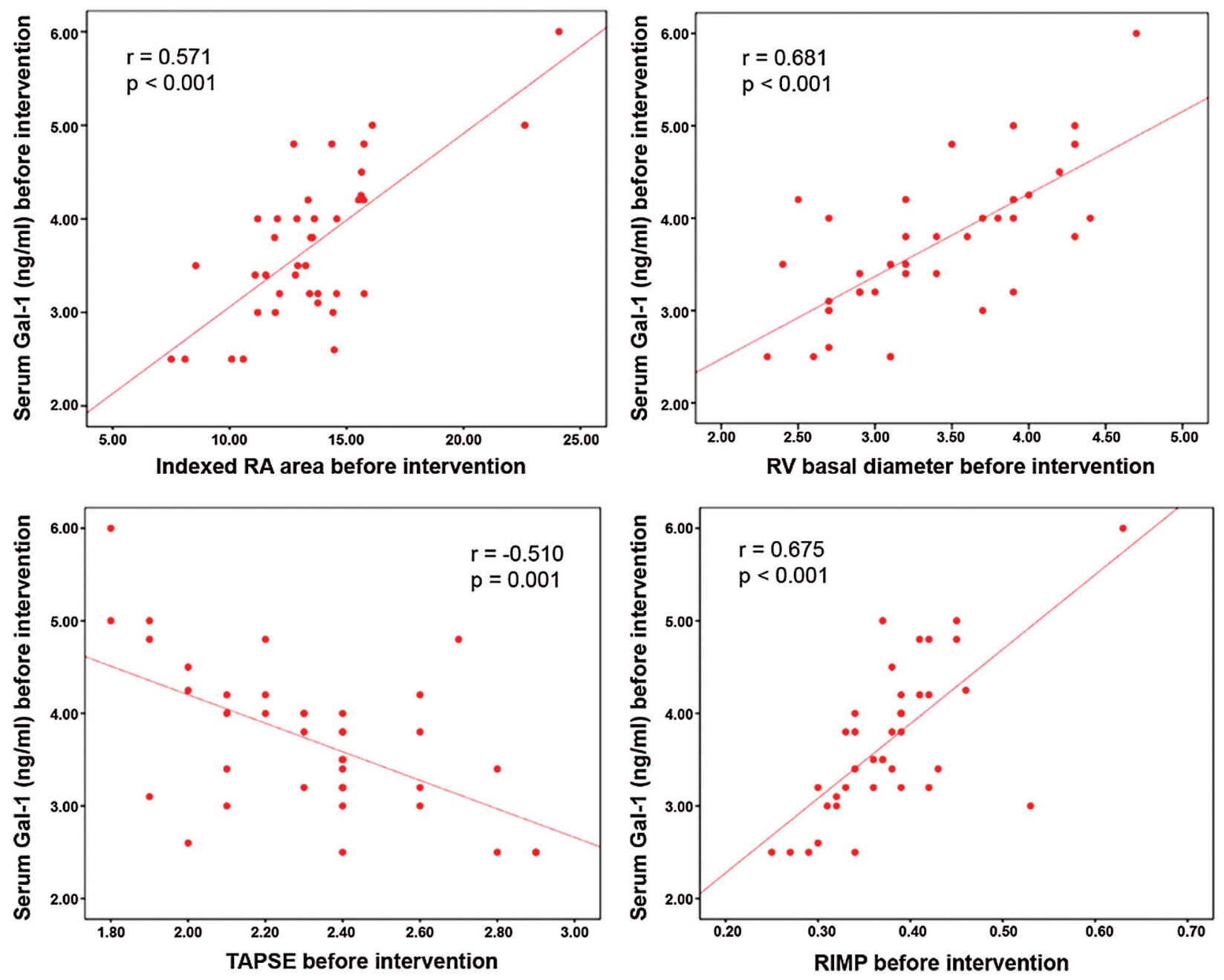

Figure 5: Spearman correlations between serum Gal-3 concentration $(\mathrm{ng} / \mathrm{ml})$ and right heart indices before intervention 


\section{Discussion}

ASD is the second most common congenital heart defect [1]. The size of the defect and the duration of shunting could determine the sequelae of ASD [21]. Although most children with ASD are asymptomatic, symptoms of dyspnea with exertion or palpitations are common in the second decade of life. Adults with ASD commonly present with symptoms including palpitations, exercise intolerance, dyspnea, peripheral edema, and cyanosis [3]. More than half of children with ASD in the present study [57.5\%] suffered from dyspnea on exertion, $32.5 \%$ suffered from palpitations, $10 \%$ developed recurring chest infection and one patient developed decompensated heart failure. A previous study demonstrated that $76.2 \%$ of children with ASD suffer from dyspnea and recurring chest infection [22].

The current study showed a significant increase in right heart indices, including indexed RA area, indexed RA volume, RVSP, RA dimensions (RA minor and major diameters), and RV dimensions (RV mid-cavity, RV longitudinal, and RV basal dimensions) before ASD closure in patients with ASD compared with the control subjects. These changes are suggestive of cardiac remodeling with considerable ASD shunts. ASDs are characterized by right-sided volume overload with a resultant progressive increase in RV and RA dimensions and progressive pulmonary vascular changes, which can be detrimental in children with ASD [7]. The mean RVSP was $43 \pm 9 \mathrm{mmHg}$ in patients with ASD. This relatively higher pulmonary systolic pressure (PSP) was consistent with another study in which the mean PSP was $40 \pm 10 \mathrm{mmHg}$ in children with ASD (mean age: 15 years) [23]. In addition, a recent study on younger children with ASD (age: $2.8 \pm 3.1$ years) showed that PSP was $27.1 \pm 7.5 \mathrm{mmHg}$ [24]. The difference in PSP could be explained by a difference in the age of patients, ASD size, and the degree of shunting, the latter of which affects pulmonary vascular changes.

By the end of the 6-month follow up period, there was a progressive improvement with a decrease in RV and RA parameters. RV dimensions showed no significant difference compared with the control group just after 1 month of closure. RA dimensions and pressure reached near the normal range after 6 months of ASD closure. Indexed RA area and RV volume were significantly decreased after closure, yet they were still significantly increased compared with the control group. These data suggest that atrial shunt closure results in a significant decrease in preload, halting or even reversing geometric heart changes. This might influence the long-term prognosis of children with ASD. However, some RA parameters might need a longer time to reach the normal range; hence, long-term follow up is indicated until all right heart indices have normalized. Similarly, previous studies have demonstrated decreased RA and RV dimensions following ASD closure [7,25-28]. Furthermore, Monfredi et al. [29] demonstrated a significant decrease in RA area and PAP in adults 6-8 weeks after percutaneous ASD closure. In addition, a significant decrease in RA volume following ASD closure was also demonstrated [7,27].

RIMP-TDI combined with TAPSE could be useful in determining the presence of RV dysfunction, particularly when evaluating chronic changes in RV function which could affect treatment decisions in patients with ASD [30]. Impairment in RV function was evident in the current study group, which was indicated by low TAPSE values and high RIMP compared with the control group. One month after ASD closure, TAPSE progressively increased and RIMP decreased, and both almost normalized with a nonsignificant difference when compared with control subjects. This is in concordance with previous studies that showed reduced TAPSE values in children with ASD [31-33], which progressively increased after percutaneous ASD closure [26,33]. In addition, other studies revealed a progressive decrease in RIMP after ASD closure [7,26,33]. Contrary to our study, higher TAPSE values and lower RIMP in patients with ASD were reported in some studies. This was described as "supernormal" RV that resulted from ASD right-sided volume overload $[25,29,34]$.

To our knowledge, serum Gal-3 concentration has not been evaluated in children after percutaneous ASD device closure. The present study supports the role of serum Gal-3 concentration as a blood 
biomarker for right-sided indices in ASD. In the present work, the mean Gal-3 concentration in control subjects was $0.63 \pm 0.13 \mathrm{ng} / \mathrm{ml}$. This value is slightly higher compared with the mean value in younger children in Mohamed et al.'s study $(0.21 \pm 0.09 \mathrm{ng} / \mathrm{ml})$ [35]. Serum Gal-3 concentration also positively correlated with age in the control group, implying that it increases with age.

Gal-3 concentration was significantly elevated in the ASD group compared with the control group (3.71 ng/ml [2.5-6 ng/ml] vs. $0.63 \mathrm{ng} / \mathrm{ml}[0.45-1 \mathrm{ng} / \mathrm{ml}]$ ). One month following ASD closure, the serum Gal-3 concentration significantly decreased; however, it did not reach the normal level compared with the control group. A high serum Gal-3 concentration with right-sided volume overload and its decrease after cessation of shunting could predict early reversal of maladaptive cardiac remodeling after ASD closure in children. Since Gal-3 is known to induce fibroblast proliferation, the decrease in serum Gal-3 concentration after ASD closure terminates further progression of myocardial fibrosis [36]. However, long-term follow up of serum Gal-3 concentration is indicated to detect complete reversal of cardiac remodeling after cessation of shunting. Few studies have demonstrated the relationship of Gal-3 concentration and congenital heart disease in children. A recent study demonstrated that Gal-3 is a promising cardiac marker for early diagnosis of heart failure in children with congenital heart disease [37]. Moreover, a higher Gal-3 concentration in children with heart failure with normal or reduced ejection fraction compared with healthy controls was observed, whereas Gal-3 concentration was slightly higher in those with a normal ejection fraction [11]. Since it is higher in children with ventricular septal defect associated with pulmonary hypertension, serum Gal-3 concentration was suggested to be a promising marker for risk stratification in such children [38]. In a recently published study that included adults with ASD, serum Gal-3 concentration was the only biomarker that decreased in response to ASD closure as an acute effect [8].

In the present study, Serum Gal-3 concentration positively correlated with ASD size, age at closure, indexed RA area, indexed RA volume, RV mid cavity diameter, RV basal diameter, RVSP, and RIMP. Serum Gal-3 concentration negatively correlated with TAPSE. With a multivariate analysis, Gal-3 concentration was highly correlated with age at closure. A higher serum Gal-3 concentration in older children with ASD might predict worsening of RA and RV structure and function and could necessitate early intervention to prevent the sequelae of right heart dysfunction.

The decline in serum Gal-3 concentration after ASD closure positively correlated with the decrease in indexed RA area, RA volume, RV mid cavity diameter, RV basal diameter, RVSP, and RIMP, and negatively correlated with TAPSE. Early defect closure would not only prevent further deterioration, but it would also improve the impairment in RA and RV dimensions and function.

The correlations between Gal-3 concentration and right-sided heart indices before and after ASD closure in children have not been previously studied. Some studies in adults show a correlation between Gal-3 concentration and some RA and RV indices in right-sided pressure overload with different etiologies.

A prospective study to detect RV structure and function in patients with pulmonary hypertension and its correlation with Gal-3 concentration and extracellular matrix (ECM) biomarkers revealed that Gal-3 concentration significantly correlated with right heart systolic function (TAPSE and RV strain) and rightsided dimensions, hypertrophy (RV mass index), and pressure (RVSP). The authors assumed that Gal-3 more specifically reflects RV myocardial extracellular matrix metabolism, since there were no differences between control subjects and patients in terms of left ventricular function [14].

Serum Gal-3 concentration correlated with interstitial fibrosis in the RA appendage and was related to $\mathrm{N}$-terminal pro-brain natriuretic peptide concentration, which is considered a marker of established cardiac stress. Thus, a high serum Gal-3 concentration might predict fibrosis and remodeling of the right atrial appendage [15]. A recent study demonstrated a higher serum Gal-3 concentration in adults with congenital heart disease with right ventricle overload. In these patients, Gal-3 concentration positively correlated with age and negatively correlated with RV longitudinal function (TAPSE and RV longitudinal strain) [39]. 
The present work suggests that serum Gal-3 concentration can be used as a biomarker of maladaptive right-sided cardiac remodeling reversal after ASD closure in children.

\section{Conclusion}

Serum Gal-3 concentration could be used as a potential biomarker for cardiac remodeling in children with ASD. Serum Gal-3 concentration was elevated in children with ASD. It then decreased significantly after percutaneous closure. Serum Gal-3 concentration correlated with age at closure, RA, and RV morphology, and function. Thus, serum Gal-3 concentration can predict progression of cardiac remodeling and could influence the decision on how best to manage children with ASD. Furthermore, serum Gal-3 concentration decreased after cessation of shunting, but it did not reach the normal range after 1 month. Moreover, its decrease correlated with the decrease in right heart indices after closure. This suggests that Gal-3 concentration can detect reversal of cardiac remodeling after closure, but long-term follow up is indicated to detect complete reversal of adverse remodeling.

\section{Study Limitations}

The sample size of the present work was small; however, the sample size was relatively larger compared with previous studies on biomarker levels in children after ASD closure. Although different echocardiographic parameters were used, three-dimensional echocardiography and speckle tracking would have allowed better evaluation of right heart structure and function. Due to limited resources, serum Gal-3 concentration was not analyzed after 6 months from ASD closure, which would have allowed better comprehension of cardiac remodeling reversal.

Acknowledgement: We would like to thank Top Edit for English language editing of this manuscript.

Ethics Approval: The committee reference number is: FMASU M D 319/2018.

Availability of Data and Materials: The data sets used and analyzed during the present study are available upon request.

Funding Statement: The authors received no specific funding for this study.

Conflicts of Interest: The authors declare that they have no conflicts of interest to report regarding the present study.

\section{References}

1. van der Linde, D., Konings, E. E., Slager, M. A., Witsenburg, M., Helbing, W. A. et al. (2011). Birth prevalence of congenital heart disease worldwide: A systematic review and meta-analysis. Journal of the American College of Cardiology, 58(21), 2241-2247.

2. van Riel, A. C., Schuuring, M. J., van Hessen, I. D., Zwinderman, A. H., Cozijnsen, L. et al. (2014). Contemporary prevalence of pulmonary arterial hypertension in adult congenital heart disease following the updated clinical classification. International Journal of Cardiology, 174(2), 299-305.

3. Geva, T., Martins, J. D., Wald, R. M. (2014). Atrial septal defects. Lancet, 383, 1921-1932.

4. Goette, A., Kalman, J. M., Aguinaga, L., Akar, J., Cabrera, J. A. et al. (2016). EHRA/HRS/APHRS/SOLAECE expert consensus on atrial cardiomyopathies: Definition, characterization, and clinical implication. Heart Rhythm, 14, e3-e40.

5. Kazmouz, S., Damien, K., Cao, Q. L., Clifford, J. K., Ziyad, M. H. (2013). Transcatheter closure of secundum atrial septal defects. Journal of Invasive Cardiology, 25(5), 257-264.

6. Jung, S. Y., Choi, J. Y. (2018). Transcatheter closure of atrial septal defect: Principles and available devices. Journal of Thoracic Disease, 10(24), S2909. 
7. Kumar, P., Sarkar, A., Kar, S. K. (2019). Assessment of ventricular function in patients of atrial septal defect by strain imaging before and after correction. Annals of Cardiac Anaesthesia, 22(1), 41-46.

8. Geenen, L. W., de Assis, L. U., Baggen, V. J., Eindhoven, J. A., Cuypers, J. A. et al. (2020). Evolution of blood biomarker levels following percutaneous atrial septal defect closure in adults. International Journal of Cardiology, Heart \& Vasculature, 30, 100582.

9. Karayannis, G., Triposkiadis, F., Skoularigis, J., Georgoulias, P., Butler, J. et al. (2013). The emerging role of Galectin-3 and ST2 in heart failure: Practical considerations and pitfalls using novel biomarkers. Current Heart Failure Reports, 10, 441-449.

10. Ibarrola, J., Sádaba, R., Garcia-Peña, A., Arrieta, V., Martinez-Martinez, E. et al. (2018). A role for fumarate hydratase in mediating oxidative effects of galectin-3 in human cardiac fibroblasts. International Journal of Cardiology, 258, 217-223.

11. Kotby, A. A., Youssef, O. I., Elmaraghy, M. O., El Sharkawy, O. S. (2017). Galectin-3 in children with chronic heart failure with normal and reduced ejection fraction: Relationship to disease severity. Pediatric Cardiology, 38(1), 95-102.

12. Calvier, L., Legchenko, E., Grimm, L., Sallmon, H., Hatch, A. et al. (2016). Galectin-3 and aldosterone as potential tandem biomarkers in pulmonary arterial hypertension. Heart, 102(5), 390-396.

13. Luo, H., Liu, B., Zhao, L., He, J., Li, T. et al. (2017). Galectin-3 mediates pulmonary vascular remodeling in hypoxiainduced pulmonary arterial hypertension. Journal of the American Society of Hypertension, 11(10), 673-683.

14. Fenster, B. E., Lasalvia, L., Schroeder, J. D., Smyser, J., Silveira, L. J. et al. (2016). Galectin-3 levels are associated with right ventricular functional and morphologic changes in pulmonary arterial hypertension. Heart and Vessels, 31(6), 939-946.

15. Hernández-Romero, D., Juan, A. V., Álvaro, L., Ana, I. R., Eva, J. et al. (2017). Galectin-3 as a marker of interstitial atrial remodeling involved in atrial fibrillation. Scientific Reports, 7(1), 1-6.

16. Lang, R. M., Luigi, P. B., Victor, M. A., Jonathan, A., Anderson, A. et al. (2015). Recommendations for cardiac chamber quantification by echocardiography in adults: An update from the American Society of Echocardiography and the European Association of Cardiovascular Imaging. European Heart Journal-Cardiovascular Imaging, 16(3), 233-271.

17. Silvestry, F. E., Meryl, S. C., Laurie, B. A., Nitin, J. B., Craig, E. F. et al. (2015). Guidelines for the echocardiographic assessment of atrial septal defect and patent foramen ovale: From the American Society of Echocardiography and Society for Cardiac Angiography and Interventions. Journal of the American Society of Echocardiography, 28(8), 910-958.

18. Rudski, L. G., Lai, W. W., Afilalo, J., Hua, L., Handschumacher, M. D. et al. (2010). Guidelines for the echocardiographic assessment of the right heart in adults: A report from the American Society of Echocardiography endorsed by the European Association of Echocardiography, a registered branch of the European Society of Cardiology, and the Canadian Society of Echocardiography. Journal of the American Society of Echocardiography: Official Publication of the American Society of Echocardiography, 23(7), 685-788.

19. Kutty, S., Ling, L., Rimsha, H., Qinghai, P., Sheela, R. et al. (2014). Systemic venous diameters, collapsibility indices, and right atrial measurements in normal pediatric subjects. Journal of the American Society of Echocardiography, 27(2), 155-162.

20. Brennan, J. M., Blair, J. E., Goonewardena, S., Ronan, A., Shah, D. et al. (2007). Reappraisal of the use of inferior vena cava for estimating right atrial pressure. Journal of the American Society of Echocardiography: Official Publication of the American Society of Echocardiography, 20(7), 857-861.

21. Rao, P. S., Harris, A. D. (2017). Recent advances in managing septal defects: Atrial septal defects. F1000Research, 6, 2042.

22. Ali, M., El-Sisi, A., El-Din, H. S., Bakhoum, S., Kandil, H. (2017). Transcatheter closure of atrial septal defect in children using the Occlutech Figulla occluder device. Al-Azhar Assiut Medical Journal, 15(1), 15-20.

23. Kaya, M. G., Baykan, A., Dogan, A., Inanc, T., Gunebakmaz, O. et al. (2010). Intermediate-term effects of transcatheter secundum atrial septal defect closure on cardiac remodeling in children and adults. Pediatric Cardiology, 31(4), 474-482. 
24. Kim, G., Kil, H. R. (2021). Changes in reverse cardiac remodeling after percutaneous atrial septal defect closure in children and adults. Congenital Heart Disease, 16(3), 211-220.

25. Akula, V. S., Durgaprasad, R., Velam, V., Kasala, L., Rodda, M. et al. (2016). Right ventricle before and after atrial septal defect device closure. Echocardiography, 33(9), 1381-1388.

26. Ozturk, O., Ozturk, U., Karahan, M. Z. (2017). Assesment of right ventricle function with speckle tracking echocardiography after the percutaneous closure of atrial septal defect. Acta Cardiologica Sinica, 33(5), 523-529.

27. Pascotto, M., Santoro, G., Cerrato, F., Caputo, S., Bigazzi, M. C. et al. (2006). Time-course of cardiac remodeling following transcatheter closure of atrial septal defect. International Journal of Cardiology, 112(3), 348-352.

28. Moradian, M., Daneshamooz, H., Shojaeifard, M., Ghadrdoost, B., Langeroudi, H. M. et al. (2018). Echocardiographic right ventricular deformation indices before and after atrial septal defect closure: A comparison between device and surgical closure. Research in Cardiovascular Medicine, 7(2), 92-97.

29. Monfredi, O., Luckie, M., Mirjafari, H., Willard, T., Buckley, H. et al. (2013). Percutaneous device closure of atrial septal defect results in very early and sustained changes of right and left heart function. International Journal of Cardiology, 167(4), 1578-1584.

30. Friesen, R. M., Schäfer, M., Burkett, D. A., Cassidy, C. J., Ivy, D. D. et al. (2018). Right Ventricular Tissue Doppler Myocardial Performance Index in children with pulmonary hypertension: Relation to invasive hemodynamics. Pediatric Cardiology, 39(1), 98-104.

31. Fetouh, R. A., Hassan, S. S., Ayda, K. A., Mohamad, I. D. (2018). Tricusped annular plane systolic excursion in children with ASD. The Egyptian Journal of Hospital Medicine, 73(10), 7761-7767.

32. Kassem, E., Humpl, T., Friedberg, M. K. (2013). Prognostic significance of 2-dimensional, M-mode, and Doppler echo indices of right ventricular function in children with pulmonary arterial hypertension. American Heart Journal, 165, 1024-1031.

33. Tosun, Ö., Ödemiş, E., Tunçer, T., Altın, H. F., Demir, H. İ. et al. (2016). Effects of atrial septal defect transcatheter closure on parameters of right ventricular functions. Turkish Journal of Thoracic and Cardiovascular Surgery, 24(4), 633-638.

34. Vitarelli, A., Sardella, G., di Roma, A., Capotosto, L., de Curtis, G. et al. (2012). Assessment of right ventricular function by three-dimensional echocardiography and myocardial strain imaging in adult atrial septal defect before and after percutaneous closure. International Journal of Cardiovascular Imaging, 28(8), 1905-1916.

35. Mohammed, L. A., Gafar, H. S., Hussien, N. R. (2014). Galectin-3 as early detector of heart failure in children with congenital acyanotic heart disease. Clinical Medicine and Diagnostics, 4(5), 90-98.

36. Yu, L., Ruifrok, W. P., Meissner, M., Bos, E. M., van Goor, H. et al. (2013). Genetic and pharmacological inhibition of galectin-3 prevents cardiac remodeling by interfering with myocardial fibrogenesis. Circulation Heart Failure, 6, 107-117.

37. Saleh, N., Khattab, A., Rizk, M., Salem, S., Abo-Haded, H. (2020). Value of Galectin-3 assay in children with heart failure secondary to congenital heart diseases: A prospective study. BMC Pediatrics, 20(1), 537.

38. Zayed, K. M., Abdelhakeem, A. M., Elhady, M., Eldahshan, T. A. (2016). Novel biomarkers for pulmonary hypertension in children with ventricular septal defect. Egyptian Pediatric Association Gazette, 64(2), 61-68.

39. Kowalik, E., Kuśmierczyk-Droszcz, B., Klisiewicz, A., Wróbel, A., Lutyńska, A. et al. (2020). Galectin-3 plasma levels in adult congenital heart disease and the pressure overloaded right ventricle: Reason matters. Biomarkers in Medicine, 14(13), 1197-1205. 